\title{
Open Heart Surgery in a Patient with Dual Chamber Pacemaker
}

\author{
Lakshmi A Sathyanarayana
}

\begin{abstract}
With changing cohort of cardiac patients, elderly patients with comorbidities like a patient with a pacemaker may be encountered in clinical practice. In patients with a pacemaker undergoing noncardiac surgery, electromagnetic interference can alter the function of a permanent pacemaker. Additional concerns during open heart surgery in a patient with a permanent pacemaker are lead displacement with venous cannulation, electrical activity during cardioplegia and damage to the device by defibrillation. Management of a patient with a pacemaker for a surgery calls for a multidisciplinary approach with involvement of cardiologist, electrophysiologist, device manufacturer, anesthesiologist, and cardiac surgeon. To the best of our knowledge, there are no case reports or guidelines about a patient with a pacemaker coming for cardiac surgery, and we report the perioperative management of a pacemaker dependent patient undergoing aortic valve replacement for severe aortic stenosis.
\end{abstract}

Keywords: Cardioplegia, Dual chamber pacemaker, Electrical interference, Open heart surgery, Pacemaker dependence, Reprogramming.

How to cite this article: Sathyanarayana LA. Open Heart Surgery in a Patient with Dual Chamber Pacemaker. Res Inno in Anesth 2018;3(2):66-69.

Source of support: Nil

Conflict of interest: None

\section{INTRODUCTION}

With changing cohort of cardiac patients, elderly patients with comorbidities like a patient with a pacemaker may be encountered in clinical practice.

In patients with a pacemaker undergoing noncardiac surgery, electromagnetic interference can alter the function of a permanent pacemaker. Additional concerns during open heart surgery in a patient with a permanent pacemaker are lead displacement with venous cannulation, electrical activity during cardioplegia and damage to the device by defibrillation. Management of a patient with a pacemaker for a surgery calls for a multidisciplinary approach with

\section{Assistant Professor}

Department of Anesthesia, Srijayadeva Institute of Cardiovascular Sciences and Research, Bengaluru, Karnataka, India

Corresponding Author: Lakshmi A Sathyanarayana, Assistant Professor, Department of Anesthesia, Srijayadeva Institute of Cardiovascular Sciences and Research, Bengaluru, Karnataka, India, e-mail: drlakshmi123@gmail.com involvement of cardiologist, electrophysiologist, device manufacturer, anesthesiologist and cardiac surgeon. ${ }^{1}$

To the best of our knowledge, there are no case reports or guidelines about a patient with a pacemaker coming for cardiac surgery, and we report the perioperative management of a pacemaker dependent patient undergoing aortic valve replacement for severe aortic stenosis.

\section{CASE REPORT}

An elderly patient aged 71 years presented with a history of breathlessness for three months. He had been inserted with a dual chamber pacemaker with rate modulation (DDDR) eight years back for syncope due to complete heart block. On precordial examination, there was grade $\mathrm{V}$ ejection systolic murmur. His ECG showed a heart rate of 70/ minute with pacing spikes preceding all the beats indicating that the patient was pacemaker dependent (A-sense V-pace) (Graph 1). Chest X-ray revealed the presence of a pulse generator in the left pre-pectoral area and leads in RA and RV (Graph 2). There was severe calcific aortic stenosis with a gradient of $90 / 53 \mathrm{~mm} \mathrm{Hg}$ across aortic valve on echocardiography. His coronary angiogram was normal.

He was scheduled for aortic valve replacement under cardiopulmonary bypass.

Preoperatively, the pacemaker function was evaluated by an electrophysiologist. On the day of surgery, the pacemaker was reprogrammed to ODO mode from DDDR by using a programmer. As the patient was pacemaker dependent, a temporary pacemaker was inserted via femoral route and VVI mode with a rate of 70 beats / min was activated. External defibrillation paddles were put on the lower back and cautery plate was positioned in the right arm. The patient was preoxygenated and induced with iv midazolam $4 \mathrm{mg}$, fentanyl $250 \mathrm{mcg}$, propofol 30 $\mathrm{mg}$, and endotracheal intubation were facilitated after injecting rocuronium $50 \mathrm{mg}$. Central venous cannulation was secured in left femoral vein to avoid pacemaker lead displacement. After heparinization, two-stage venous cannulation through the right atrium and aortic cannulation were done, and cardiopulmonary bypass was initiated. After applying aortic cross-clamp, ante-grade blood cardioplegia was given through aortic root line and the temporary pacemaker was switched off. Aortic valve replacement with $23 \mathrm{~mm}$ bioprosthetic valve was performed. Thorough deairing was done taking precaution to avoid pacemaker lead displacement. Cross-clamp was removed. Total 


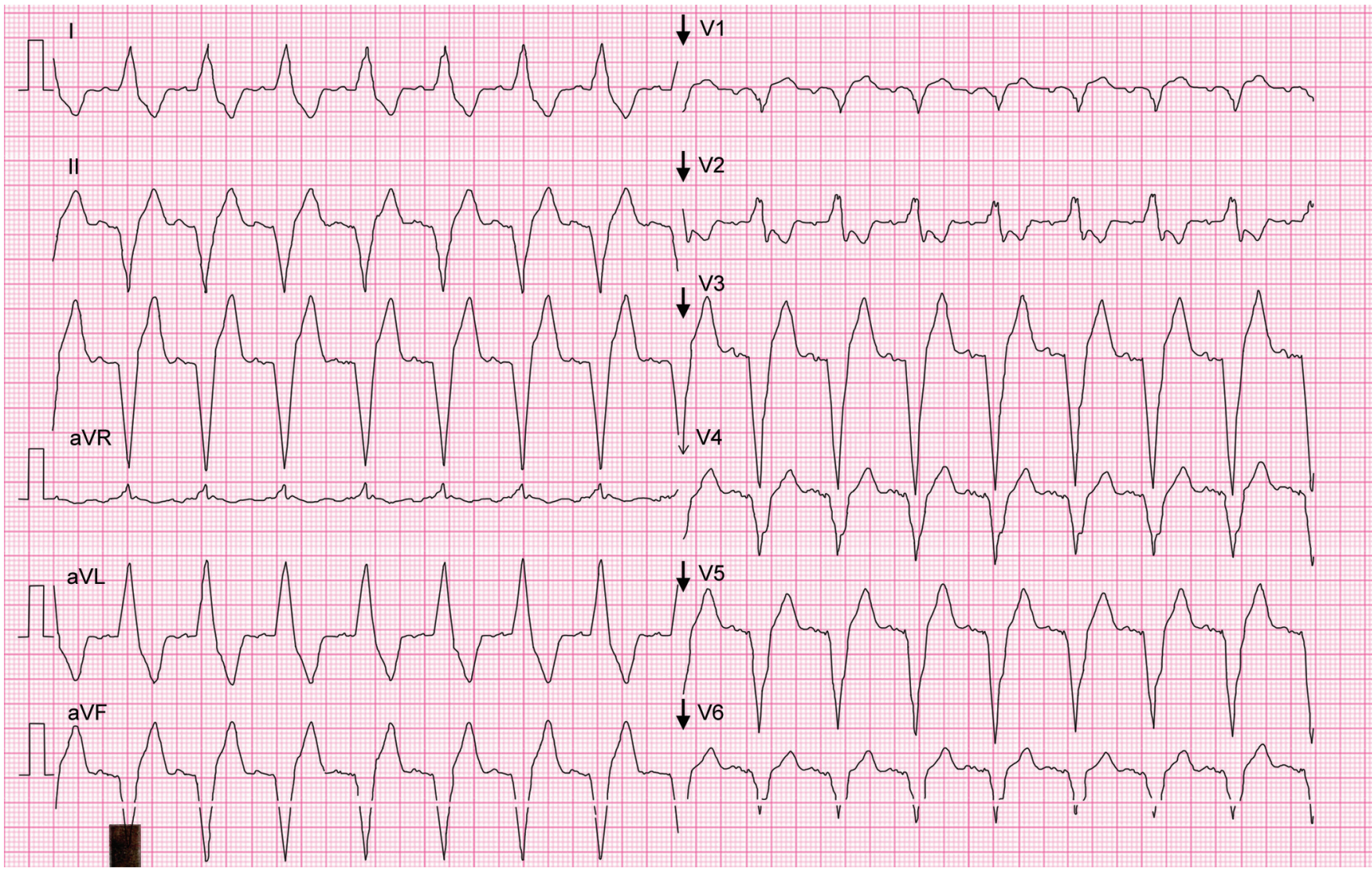

Graph 1: ECG: rate 100 beats/minute atrial sensing and ventricular pacing, LBBB morphology, superior axis, suggesting right ventricular apical pacing

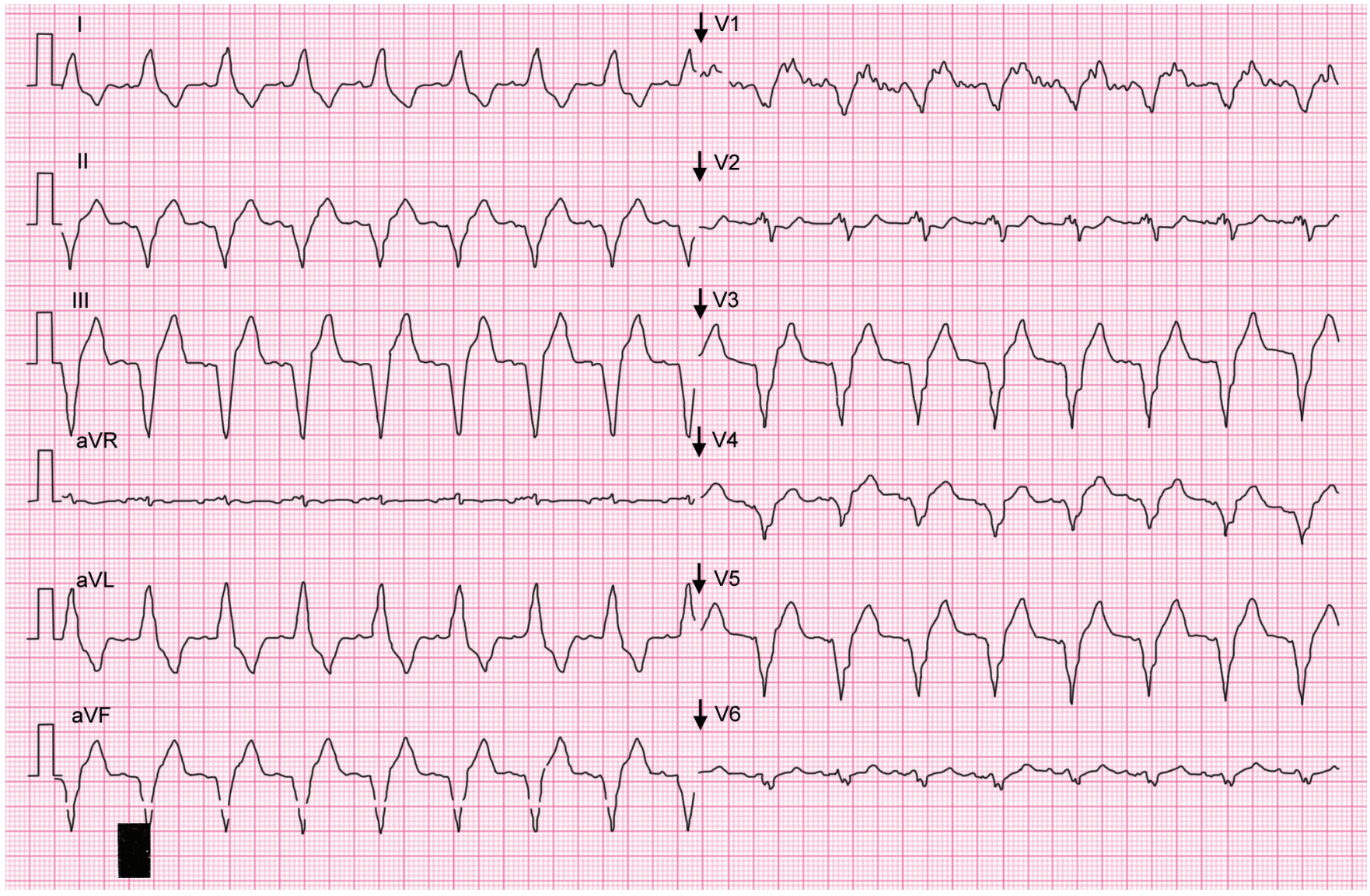

Graph 2: X-ray chest: PA view, postoperative status, pulse generator in right pre-pectoral area with leads in the right atrium and right ventricle 
bypass time was $147 \mathrm{~min}$ and cross-clamp time was 90 min. During rewarming, injection amiodarone $300 \mathrm{mg}$ was given as prophylaxis against ventricular fibrillation. Electrocautery was used in short bursts intermittently throughout the procedure, which used to cause inhibition of pacer. Inadequate electrical activity of 20 beats / minute necessitated temporary pacing at 75 beats/minute in VVI mode through temporary pacing. The patient was weaned from cardiopulmonary bypass with $5 \mathrm{mcg} / \mathrm{kg} / \mathrm{minute}$ of dobutamine. Oxygenation status and electrolyte balance were monitored throughout the perioperative period and maintained within normal limits. The patient was extubated on the first postoperative day and inotropic support was weaned off. A permanent pacemaker was reset to DDD mode with the help of a programmer. Pacemaker function was reassessed and reset by the electrophysiologist using a programmer before discharge of the patient two weeks later.

\section{DISCUSSION}

Perioperative management of a patient with permanent pacemaker involves the care of the device as well as anesthetic implications of the device. Sensing demand pacemakers are commonly used for patients requiring a permanent pacemaker. Dual chamber pacemakers preserve the normal atrioventricular contraction sequence and are beneficial in patients, where atrial contraction is important for ventricular filling (e.g., aortic stenosis). ${ }^{1}$

Rate responsive pacemakers can sense and react to the changing needs of the heart, but they also react to other noncardiac electromagnetic signals. Electromagnetic interference increases with increasing number of leads and with rate responsiveness.

Electromagnetic interference (EMI) is common during surgery and can alter the function of a demand pacemaker. Electrocautery and defibrillation are the sources of EMI which can cause different alterations in the functions of the device. Electrocautery is used to cut or coagulate tissue and it involves passing a high voltage, high frequency $(10,000 \mathrm{~Hz})$ current. This can cause device malfunction. If the electrocautery is activated before it is in contact with the instrument used to cauterize the tissue, the current can arc through the air and demodulate to a frequency range that is sensed by the device. Then the device responds inappropriately to this current. ${ }^{1}$

Another concern during open heart surgery is the use of defibrillators. Defibrillators deliver high voltage current in the vicinity of the heart. Although pacemakers and internal cardioverter defibrillators (ICD) employ Zener diodes to shunt high levels of current away from the delicate internal circuitry, repeated attempts at defibrillation can overwhelm these circuits leading to permanent device malfunction. Also, current shunted away from the device can cause burns at the lead-tissue interface and cause loss of capture. ${ }^{2}$
In patients with aortic stenosis due to myocardial hypertrophy, inadequate myocardial protection and occurrence of atrial and ventricular arrhythmias are common which may require repeated attempts of defibrillation. In our patient, periodic infusion of cold blood cardioplegia and amiodarone injection during rewarming was done. Our patient did not require cardioversion or defibrillation throughout the procedure.

A third concern about pacemakers is during cardioplegic arrest. The pacemaker has to be inactivated to sensing mode during cardioplegia to guide in assessing return of intrinsic electrical activity and adequacy of myocardial protection.

Preoperative evaluation of a patient with permanent of the pacemaker has to be individualized to the patient, type of the device and the procedure being performed. Symptomatic bradycardia or syncope or history of AV nodal ablation suggest that the patient may be dependent on the pacemaker. Symptoms which lead to pacemaker insertion and resolution of the symptoms after insertion has to be assessed. Significant comorbid conditions like heart failure have to be optimized..$^{3-5}$

Physical examination, relevant medical records, and chest X-ray are to be reviewed. ECG helps in identifying the underlying rate, rhythm, and dependence on the pacemaker device (Fig. 1). Our patient had pacemaker spike before every beat indicating pacemaker dependency. Multidisciplinary communication and team approach are required throughout the perioperative period. Device manufacturer provides the patient with a card which contains the model number, serial number, date of implant, reason for implant and implanting hospital and follow-up hospital. Surgical team has to inform the electrophysiologist about the type of surgery, device position, patient position during the procedure, type of electrocautery to be used, other sources of EMI likely to be present, whether cardioversion or defibrillation will be required, operation in close proximity of the device, availability

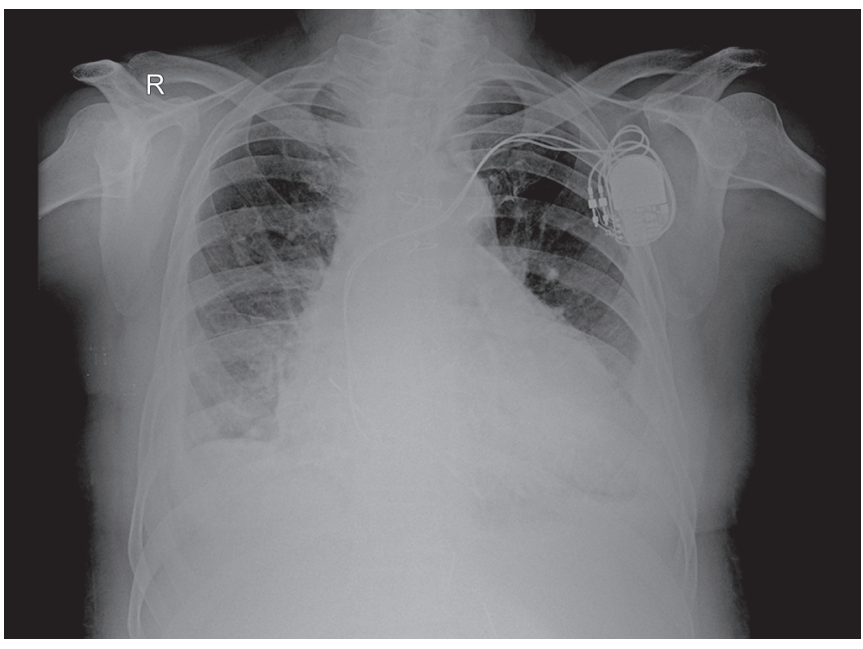

Fig. 1: Chest X-ray with pacemaker device 
of the programmer and industry employed allied health professional. Electrophysiologist provides information about the indication for the implant, the extent of any congestive heart failure, dependency on the pacemaker, the complexity of the device and if the device is approaching replacement. ${ }^{4,5}$

Reprogramming of the device is required for any major procedure in thorax or abdomen, surgery with significant EMI, rate enhancements present should be disabled, as positive pressure ventilation or use of depolarising muscle relaxant can lead to inappropriate rate responsiveness. Reprogramming to an asynchronous mode with a rate higher than the underlying rate ensures that no over sensing or under-sensing from EMI will occur. But reprogramming will not protect the device from internal damage or reset caused by EMI. ${ }^{4,5,6}$

In our patient, ODO mode with backup temporary VVI pacing was selected as the patient was pacemaker dependent and it was desirable to have electrical quiescence during cardioplegic arrest, and electrical quiescence is possible with ODO.

Bipolar lead sensing is more resistant to oversensing from muscle artifact or stray electromagnetic fields. Our patient had bipolar leads in both atria and ventricle. We used monopolar cautery taking care that the return electrode is positioned away from and the circuit is in the transverse plane. Short bursts of energy $(<5 \mathrm{sec})$ was used intermittently.

Intraoperatively backup source of pacing, defibrillation, are to be kept ready. Vigilance and monitoring to detect hemodynamic compromise are done by ECG, arterial blood pressure and pulse oximetry. Some ECG monitors permit filtering of pacemaker spikes, and such filtering should be disabled. Frequent ABGs to detect any electrolyte/acid-base abnormalities are to be done.

To the best of our knowledge, clear data or literature guiding central venous catheter insertion (CVC)/venous cannulation for cardiopulmonary bypass are lacking. ${ }^{7}$ Our patient had permanent pacemaker lead placed 6 years ago, and temporary pacing lead placed on the day of surgery. And as the patient was pacemaker dependent, we used femoral access for CVC placement. Though two stages venous cannula in right atrium was used for $\mathrm{CPB}$.

There are incidents and case reports of permanent pacemaker lead displacement, especially recently inserted leads during removal of the temporary pacing lead and chest clapping and intense physiotherapy. ${ }^{7}$ Constant vigilance and continuous monitoring throughout the perioperative period is necessary. If a pacemaker fails, measures to be taken are the availability of drugs like isoprenaline, atropine, epinephrine, transcutaneous pacing or transvenous pacing or epicardial leads and meantime early and effective cardiopulmonary resuscitation is required till the rhythm is restored. ${ }^{2}$
If the patient develops VT/VF, cardioversion or defibrillation can be done with required doses of biphasic shocks. Pacer should be turned to VOO/AOO mode during cardioversion, efforts to minimize the current flow through the pulse generator and leads, with external paddles as far away from a pulse generator and perpendicular to the major axis of the pulse generator and lead system assembly. ${ }^{1,4}$

Though anesthetic agents cause no change in pacing thresholds, factors affecting pacing threshold during anesthesia are hyperventilation, acid-base or electrolyte disturbances, significant volume loads, blood transfusion, myocardial ischemia, and high blood concentrations of local anesthetics, can all increase pacing threshold. 1,2,4

Postoperatively, vigilant monitoring, immediate availability of backup pacing, defibrillation, or both are required. Pacemaker settings are to be restored. Formal interrogation of the pacemaker has to be done as appropriate. ${ }^{4,5}$

\section{SUMMARY}

Open heart surgery can be safely conducted in a pacemaker dependent patient by taking care of the device, by reprogramming the generator to a sensing mode, use of a temporary transvenous pacemaker and minimizing defibrillation and by postoperative resetting. Patient has to be monitored throughout the perioperative period with the maintenance of oxygenation and electrolyte balance and keeping all emergency drugs and pacing and defibrillatory equipments readily available. Calls for expert opinions and guidelines about central venous cannulation and perioperative management of a pacemaker dependent patient for cardiac surgery.

\section{REFERENCES}

1. Madigan JD, Choudhri AF, Chen J, Spotnitz HM, Oz MC, Edwards N. Surgical management of the patient with an implanted cardiac device: implications of electromagnetic interference. Annals of Surgery 1999;230(5):639.

2. Gallagher MD, David Hayes MD, Jane EH. Practice advisory for the perioperative management of patients with cardiac implantable electronic devices: pacemakers and implantable cardioverter-defibrillators. Anesthesiology 2011;114(2):247-261.

3. Rapsang AG, Bhattacharyya P. Pacemakers and implantable cardioverter defibrillators-general and anesthetic considerations. Revista Brasileira de Anestesiologia. 2014;64(3):205-214.

4. Rastogi S, Goel S, Tempe DK, et al. Anaesthetic management of patients with cardiac pacemakers and defibrillators for noncardiac surgery. Annals of Cardiac Anaesthesia 2005;8(1):21-32.

5. Senthuran S, Toff WD, Vuylsteke A, et al. Editorial III. British Journal of Anaesthesia [Internet]. Elsevier BV 2002;88(5):627-631.

6. Andersen C, Madsen GM. Rate-responsive pacemakers and anaesthesia A consideration of possible implications. Anaesthesia. 1990;45(6):472-476.

7. Sharma P, Singh MK, Vats A, et al. Central Venous Catheter Insertion in Patients With Transvenous Pacemaker or Defibrillator Leads. Journal of Cardiothoracic and Vascular Anesthesia 2011;25(4):e25. 